\title{
Cid Kaoui Saïd
}

\section{O. Ould Braham}

\section{OpenEdition}

Journals

Édition électronique

URL : http://journals.openedition.org/encyclopedieberbere/2277

DOI : 10.4000/encyclopedieberbere.2277

ISSN : 2262-7197

\section{Éditeur}

Peeters Publishers

\section{Édition imprimée}

Date de publication : 1 février 1994

Pagination : 1951-1953

ISBN : 2-85744-696-9

ISSN : 1015-7344

\section{Référence électronique}

O. Ould Braham, « Cid Kaoui Saïd », Encyclopédie berbère [En ligne], 13| 1994, document C62, mis en ligne le 01 mars 2012, consulté le 25 septembre 2020. URL : http://journals.openedition.org/ encyclopedieberbere/2277 ; DOI : https://doi.org/10.4000/encyclopedieberbere.2277

Ce document a été généré automatiquement le 25 septembre 2020

(c) Tous droits réservés 


\section{Cid Kaoui Saïd}

\section{O. Ould Braham}

1 Berbérisant algérien, auteur de dictionnaires, Saïd ben Mohammed-Akli Cid Kaoui est né le 12 mars 1859 à Ahammam, village de la puissante tribu des Oulad Abd el Djebar et situé dans la wilaya de Bejaïa près de Oued Amizour. Si sa mère, Chérifa bent Saïd ben Ahmed est née dans ce même secteur, au village de Taourirt, son père, Mohammed Akli (Muḥend Akli), lui, était originaire des Beni Sedka (At Sedqa), tribu de la Kabylie du Djurdjura, et vint s'établir dans cette zone de l'Oued Sahel après la conquête de la Kabylie par l'armée française en 1856-57. Est-ce pour y remplir des fonctions de notable, nommé ainsi par la puissance occupante? Aucune information à ce sujet. Un lettré en langue arabe, c'est certain, qui dut appartenir à la caste maraboutique, témoin l'extrait d'acte judiciaire en date du 9 avril 1887 où il fut fait mention «du jeune Si Essaïd (lire Si Saïd), fils de feu Mohammed Akli Cid Kaoui ». Chacun sait que le titre de « $\mathrm{Si}$ » est réservé exclusivement dans ces régions aux marabouts et exceptionnellement aux hommes versés dans la science religieuse.

2 Dans les milieux lettrés musulmans, le père de notre lexicographe dut se faire appeler Muḥammad 'Aklî as-Sadqâwî, et cette nisba servit de nom patronymique au fils quand celui-ci, encore jeune, endossa la tenue militaire. Il se fit en effet enregistrer dans les spahis sous ce vocable mais avec la fantaisie orthographe que l'on connait. Durant le conflit franco-prussien de 1870, Mohammed Akli se porta comme engagé volontaire pour la durée de la guerre sur le front de Sedan où il reçut plusieurs blessures. Mourutil peu de temps après? Cela ne fait pas de doute. À sa majorité, Saïd Cid Kaoui avait perdu ses deux parents comme il est indiqué dans l'extrait d'acte précité.

3 Peu d'informations sur l'enfance et les premières années de jeunesse de notre auteur. Il fréquenta, comme les quelques très rares autochtones de son rang, l'école primaire française de la ville de Bougie, parallèlement à l'étude et la lecture du Coran dans l'école traditionnelle du quartier, avant d'entrer au lycée franco-arabe de Constantine, où il fut doté d'une solide instruction en français et en arabe. Ce qui lui ouvrira, plus tard, les portes de la fonction d'interprète militaire.

Avant d'embrasser la carrière en question, à l'âge de 18 ans, il s'enrôla dans les spahis comme engagé volontaire pour une durée de quatre années, avec le grade de brigadier 
puis celui de maréchal des logis. Libéré de ses fonctions le 5 mars 1881, il obtint une place de surveillant au lycée d'Alger, avant de se rengager dans le $1^{\text {er }}$ Régiment de Spahis le 13 juillet 1882 pour quatre nouvelles années. Vers la rentrée de 1880, il s'inscrivit à l'Université d'Alger, en médecine, études qu'il poursuivit pendant deux ans avant d'opter pour un cours d'interprète. Après quoi, ayant réussi ses examens avec succès, il fut recruté le 26 septembre 1886 dans le corps des interprètes militaires. En tant qu'auxiliaire de $2^{\mathrm{e}}$ classe, il exerça successivement auprès du Commandant supérieur de Boghar (1886-1888), au Bureau arabe de Ouargla (1888), au Bureau arabe de Boghar (1888-1890), au Bureau arabe de Ghardaïa (1890-1891) et à la subdivision de Dellys (1891-1895). Ce n'est qu'à ce dernier poste qu'il fut promu interprète militaire auxiliaire de $1^{\mathrm{e}}$ classe et affecté ensuite à la subdivision de Laghouat (1895) et au Bureau arabe de Chellala (1896-1905) où il devint officier interprète de $1^{\mathrm{e}}$ classe, dans le grade de capitaine. Durant les années 1905-1906, il est affecté à la section des affaires indigènes de la Division d'Alger avant de rejoindre le cercle de Bou Sâada, le 6 septembre 1906. Deux années plus tard, le 20 octobre, il fut admis sur sa demande à faire valoir ses droits à la retraite, après plus de trente ans de services.

Cid Kaoui s'est marié en 1889 avec une Française d'Algérie, Mlle Léonie Richebois, née en 1868 à L'Arba dans la Mitidja, domiciliée à Bordj Menaïel et fille d'un brigadier de gendarmerie en retraite. Il a obtenu sa naturalisation par décret du 27 janvier 1890. Il eut trois enfants issus de ce mariage: Léon, né à Ghardaïa en 1890 (mort jeune), Marguerite, née à Dellys en 1892 (décédée en 1978 dans les Hautes-Alpes; a quitté l'Algérie en 1963), Baya-Lucie, née en 1904 (morte de maladie l'année de la mort de son père).

6 Après son passage à l'Université et lors de sa préparation à une carrière dans le corps des interprètes militaires, il fut désigné en 1884 en qualité de juré aux examens de berbère. C'est à partir de cette date, pour répondre à la demande des candidats, qu'il songea à réaliser un dictionnaire kabyle, voulant ainsi dépasser les tentatives des Pères Creuzat (1873) et Olivier (1878). Ce projet qu'il mena simultanément avec la rédaction d'un autre dictionnaire consacré au touareg ne fut pas mené à bonne fin, tel qu'il nous le rapporte lui-même: "Au commencement de 1887 je travaillais à un dictionnaire français-kabyle, lorsque je fus nommé au poste de Ouargla. Avant cette époque, j'avais entrepris déjà de réunir les matériaux nécessaires pour composer un dictionnaire français-tamâhaq ; mais, jusque là, je n'avais pu rassembler qu'un très petit nombre de mots. À mon arrivée à Ouargla, j'entrai en relations avec des Indigènes d'In-Salah connaissant parfaitement la langue tamâhaq, et qui étaient, en même temps, lettrés en langue arabe. » (Avant-propos, Dictionnaire français-tamâhaq).

7 Il publia en 1894 son Dictionnaire français-tamâhaq, travail déjà achevé en 1890 mais empêché de voir le jour promptement : retardé par les lenteurs des commissions de publications. Six ans plus tard, c'est le Dictionnaire pratique tamâhaq-français qui parut en librairie; il est l'abrégé du premier dictionnaire. Il s'intéressa ensuite aux parlers berbères du Maroc Central et du Sud-ouest marocain auxquels il consacra un nouvel ouvrage, le Dictionnaire français-tachelh'it et tamazir't (1907). Les appréciations continuellement critiques de René Basset, une personne de référence dans le domaine des études berbères de l'époque, ont fait sortir notre auteur de ses gonds, ce qui a donné lieu à l'impression successive de trois brochures - dans lesquelles il essaya d'apporter des arguments pour montrer la justesse de ses vues - qui sont dans l'ordre : À Monsieur René Basset. Réponse à une critique littéraire, 11 p., s.d (1908) : À Monsieur René 
Basset. Réponse à une critique littéraire (suite), 11 p., s.l, s.d (1908) : Étude comparative entre deux dictionnaires français-touareg, publiés respectivement en 1894 et en 1908, 13 p., s.l., s.d (1909).

8 Membre de la Société historique algérienne - éditrice de la Revue Africaine -depuis environ 1896, juré aux examens des primes et diplômes de berbère depuis 1884 et ayant accompli une carrière exemplaire, il fut honoré de plusieurs hautes distinctions: Officier du Nichan Iftikhar (1895), Officier d'Académie (1905) et Chevalier de la Légion d'honneur (1904); il reçut en outre, lors de son passage à Paris, une médaille de bronze à l'Exposition Universelle de 1900 couronnant ses deux dictionnaires touareg.

Il décéda le 15 décembre 1910 à Bordj Menaïel où il s'était retiré avec sa famille.

\section{BIBLIOGRAPHIE}

\section{Sources}

Archives du Gouverneur Général d'Algérie, série H (Aix-en-Provence).

Fonds Instruction Publique (Paris).

Fonds Légion d'honneur (Paris).

Études

CID KAOUI S., «Étude comparative entre deux dictionnaires français-touareg publiés respectivement en 1894 et en 1908 ", Études et Documents Berbères, n 4, 1988, p. 32-48, Paris.

OULD-BRAHAM 0., "Quelques documents autour de l'édition du premier dictionnaire de Cid Kaoui », Études et Documents Berbères, nº 2, 1987, p. 156-162, Paris.

INDEX

Mots-clés : Histoire contemporaine 